\title{
Определение высококипящих органических соединений с использованием блочно-порозных сорбционных систем
}

\author{
(C) 2021 Платонов И.А., Новикова Е.А., Карсункина А.С., \\ Лебедев А.Н., Тупикова Е.Н. \\ Самарский национальный исследовательский университет им. академика С.П. Королева, Самара
}

Поступила в редакцию 21.06.2021 г.

DOI: $10.17308 /$ sorpchrom.2021.21/3630

Определение микропримесей токсичных веществ в атмосферном воздухе на уровне концентраций 0.1-0.5 мг/м ${ }^{3}$ затрудняется стадией концентрирования, которая является одним из основных источников погрешности. Концентрирование осуществляется с использованием сорбентов различной природы. Блочный материал с варьируемой порозностью, разработанный в Самарском Университете (ранее КуАИ), обладает преимуществами по сравнению с другими сорбентами, вследствие возможности пропускания большого объема воздуха и простоты десорбции. Целью работы являлось изучение возможности использования блочного материала с варьируемой порозностью в качестве основы для сорбционной системы, предназначенной для концентрирования высококипящих органических соединений.

Для изготовления экспериментальных образцов использовалась сталь марки Х18Н10Т. Процесс изготовления образов включал стадии формирования блоков основы, травления образцов в соляной кислоте с дальнейшим оксидированием при температурах 350,500 и $750^{\circ} \mathrm{C}-$ цвет поверхности становился также более тёмным: чем больше температура, тем темнее. Исследование полученных образов проводили на примере определения высококипящего соединения - диоктилфталата (ДОФ). Для исследования полученных образцов, а также для сравнительной оценки абсорбционного (система концентрирования - поглотитель с изопропиловым спиртом) и адсорбционного (система концентрирования - силиконовая трубка с образцом сорбционном системы) концентрирования, была собрана установка, позволяющая получать газовые потоки, содержащие микропримеси ДОФ в воздухе. Количественный анализ полученных концентратов проводили методом газовой хроматографии.

В результате количественного анализа экстракта ДОФ в изопропиловом спирте (ИПС), полученного путём абсорбционного концентрирования, определили ориентировочную концентрацию ДОФ в газовой фазе $\mathrm{C}=0.46 \pm 0.15 \mathrm{~m} / \mathrm{m}^{3}$. Установлено, что наименьшее количество ДОФ десорбировано с необработанного образца. Это связано с тем, что данный образец практически не обладает высокоразвитым адсорбционным слоем. Количество десорбированного ДОФ с образцов, обработанных при разных температурах, отличается незначительно. В рамках работы проведена сравнительная оценка различных способов концентрирования. Установлено, что стандартизированная методика определения ДОФ с абсорбционным концентрированием дает явно заниженные результаты по сравнению с адсорбционным концентрированием. Это может быть связано как с неполным улавливанием ДОФ из газового потока, так и с внесением дополнительных погрешностей при упаривании экстракта. Адсорбционное концентрирование с использованием активированного угля также даёт заниженный результат. Десорбция ДОФ с активированного угля проводилась также, как и в случае с полученными экспериментальными образцами. Заниженный результат может быть связан с неполнотой десорбции ДОФ с активированного угля. Таким образом, использование предлагаемых систем позволяет повысить не только правильность определения ДОФ за счет более эффективного улавливания компонента, но и прецизионность анализа.

Ключевые слова: сорбционное концентрирование, высококипящие органические соединения, пробоподготовка, исследование газовых сред, хроматографический метод анализа, блочнопорозные системы. 


\section{Введение}

Одной из актуальнейших задач аналитической химии является определение микропримесей в воздушных средах, таких как атмосферный воздух, воздух рабочей зоны и жилых помещений, выбросы промышленных предприятий. Например, для обеспечения экологической безопасности производств полимерных композиционных материалов и изделий на их основе, а также в целях проведения оценки безопасности использования получаемых изделий в быту необходимо проводить определение пластификаторов в воздухе на уровне концентрации 0.1-0.5 мг/ $\mathrm{m}^{3}$. Такой анализ невозможен без проведения концентрирования, что является одним из основных источником погрешностей при проведении анализа. Для концентрирования органических микропримесей из газовых сред широко используются сорбенты различной природы, но их использование характеризуется высоким газодинамическим сопротивлением слоя сорбента, что является немаловажным сдерживающим фактором при необходимости пропускания больших объемов воздуха, а также трудности проведения десорбции, особенно, высококипящих органических соединений.

В Самарском Университете (ранее КуАИ) в 1960-е годы под руководством профессора А.М. Сойфера был создан блочный материал с варьируемой порозностью, известный как «металлорезина». Данный материал широко используется в качестве демпфирующего материала в габаритных механизмах, фильтров, подшипников скольжения, виброизоляторов и упругопористых элементов, а также как носитель для микро- и наночастиц катализаторов [1-3]. Следует отметить, что возможность варьирования порозности и, как следствие, газодинамического сопротивления слоя материала, а также возможность формирования сорбционных слоев различного строения при сохранении площади поверхности матери- ала делает перспективным его применение в качестве основы для сорбционных систем.

Целью работы являлось изучение возможности использования блочного материала с варьируемой порозностью в качестве основы для сорбционной системы, предназначенной для концентрирования высококипящих органических соединений.

\section{Экспериментальная часть}

Для изготовления экспериментальных образцов использовалась сталь марки Х18Н10Т. Процесс изготовления образов включал следующие этапы:

1 этап - формирование блоков. Для формирования блоков основы брали предварительно рассчитанную навеску проволоки. Навитую и растянутую проволоку укладывали и сворачивали таким образом, чтобы заготовка поместилась в пресс-форму, затем прессовали путем воздействия пуансонов до определённого значения высоты. Таким образом, были получены блоки диаметром 8 мм и высотой 5 мм, порозность полученных образца равна 0,7 . После формирования осуществлялось обезжиривание образцов при помощи изопропилового спирта в ультразвуковой ванне в течение 2 минут и последующее промывание блоков потоком дистиллированной воды для удаления остаточных загрязнений.

2 этап - травление. Травление блока осуществляли в 20\%-ном растворе соляной кислоты в течение 2 минут при температуре $70^{\circ} \mathrm{C}$, затем блоки многократно промывали дистиллированной водой и обрабатывали ультразвуком частотой 18 кГц в течение 1-2 мин. на установке УЗУ -0.25 .

3 этап - оксидирование. Оксидирование проводилось при температурах $350^{\circ} \mathrm{C}$ и $500^{\circ} \mathrm{C}$ в течение 3 часов и при $750^{\circ} \mathrm{C}$ в течение 1 часа. Затем оксидированный образец помещали в дистиллированную воду и обрабатывали ультразвуком частотой 18 кГц в течение 2-3 мин для удаления окалины и рыхлых 
слоев, плохо сцепленных с поверхностью образца.

Визуально образование оксидного слоя на поверхности образцов при травлении проявлялось в виде более тёмного оттенка поверхности. При последующем оксидировании образцов при разных температурах цвет поверхности становился также более тёмным: чем больше температура, тем темнее.

Исследование полученных образов проводили на примере определения высококипящего соединения - диоктилфталата (ДОФ). Данное соединение является представителем пластификаторов, широко используемых при получении пластмасс и изделий на их основе, в частности, линолеума.

Для исследования полученных образцов, а также для сравнительной оценки абсорбционного и адсорбционного концентрирования была собрана установка, позволяющая получать газовые потоки, содержащие микропримеси ДОФ в воздухе (рис. 1). Для получения парогазовых смесей, содержащих летучие органические и неорганические соединения, существует множество способов [4-8], однако, пластификаторы, в том числе ДОФ, отличаются малой летучестью, что существенно затрудняет процесс. Для создания газового потока использовался поглотитель Рыхтера, заполненный чистым ДОФ и помещенный в водяной термостат при температуре $60^{\circ} \mathrm{C}$, через поглотитель проходил поток воздуха со скоростью $0.5 \mathrm{~cm}^{3} /$ мин.

Для проведения абсорбционного концентрирования [9] в качестве системы концентрирования был взят поглотитель, заполненный изопропиловым спиртом объёмом $5 \mathrm{~cm}^{3}$. Газовый поток, содержащий микропримеси ДОФ, пропускали через поглотитель Рыхтера с ИПС, затем полученные экстракты упаривали до объёма $2 \mathrm{~cm}^{3}$. При адсорбционном концентрировании в качестве системы концентрирования использовалась силиконовая трубка, в которую помещали образец сорбционной системы. Также в рамках работы проводили сравнительную оценку сорбционных свойств, полученных образцов и традиционного используемого для проведения концентрирования сорбента - активированного угля БАУ. В этом случае в качестве системы концентрирования выступала изогнутая стеклянная трубка, наполненная сорбентом. Извлечение ДОФ с образцов и активированного угля проводилось с использованием изопропилового спирта в ультразвуковой ванне в течение 2 минут.

Количественный анализ полученных концентратов проводили методом газовой хроматографии на хроматографе «Кристалл 5000.1» на кварцевой капиллярной колонке 8 м $\times 0.53$ мм с неподвижной фазой SE-30 с использованием пламенно-ионизационного детектора.

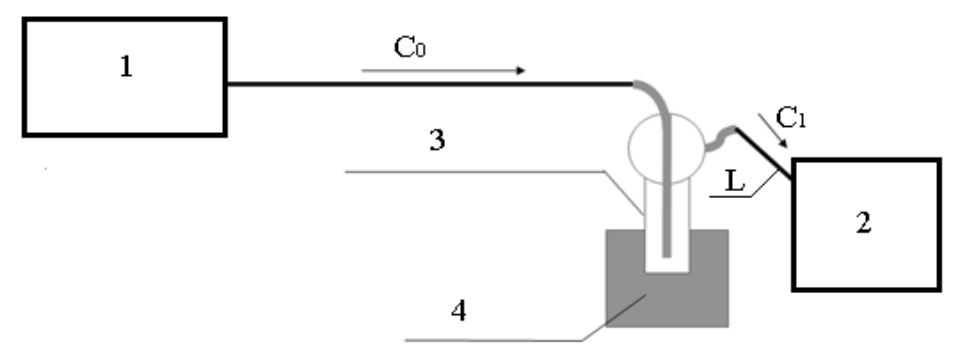

Рис. 1. Схема установки для проведения концентрирования

1 - компрессор с регулятором расхода газа; 2 - система концентрирования;

3 - поглотитель Рыхтера заполненный ДОФ; 4 - водяной термостат; $\mathrm{C}_{0}$ - поток воздуха,

$\mathrm{C}_{1}$ - поток воздуха с микропримесями ДОФ; $\mathrm{L}$ - силиконовая трубка

Fig. 1 Diagram of the installation for the concentration process.

1 - compressor with gas flow regulator; 2 - concentration system; 3 - Richter absorber

filled with DOP; 4 - water thermostat; $\mathrm{C}_{0}-$ air flow, $\mathrm{C}_{1}$ - air flow with trace amounts of DOP; $\mathrm{L}$ - silicone tube 


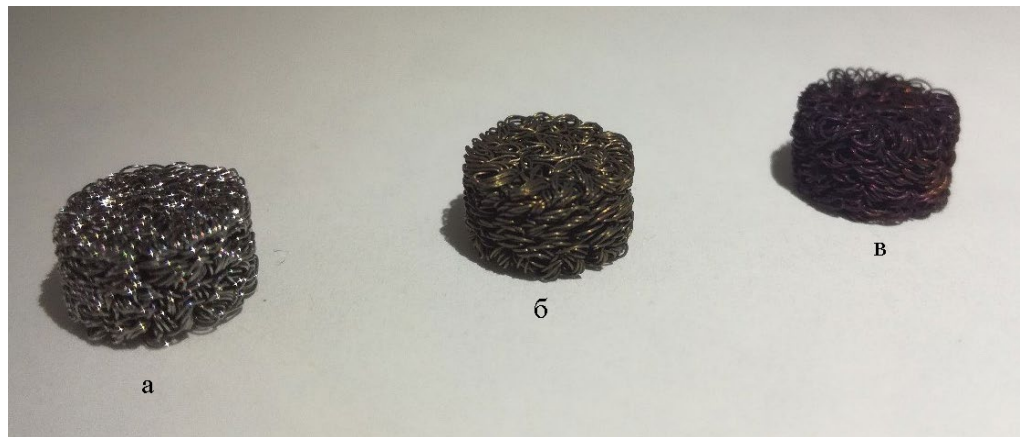

Рис. 2. Фотографии экспериментальных образцов блочного материал с варьируемой порозностью: a - с необработанной поверхностью, б - с поверхность, подвергнутой травле-

нию; в - с поверхностью, подвергнутой травлению и оксидирвоанию при $500^{\circ} \mathrm{C}$

Fig. 2 Photographs of experimental samples of the block material with variable porosity: $\mathrm{a}$ - with untreated surface, $\mathrm{b}$ - with etched surface; $\mathrm{c}$ - with a surface etched and oxidised at $500{ }^{\circ} \mathrm{C}$

\section{Обсуждение результатов}

При реализации методик, описанных выше, была получена серия экспериментальных образов сорбционного материала (рис. 2).

На поверхности образцов с необработанной поверхностью наблюдается относительно гладкая поверхность с редкими порами и неровностями (рис.3а). На образцах, которые подвергали термической обработке можно увидеть неоднородную поверхность, причем наблюдается образование не только пор и трещин, но и сферических частиц диаметром 0.3-1 мкм (рис.3б). Визуально образование оксидного слоя на поверхности об-

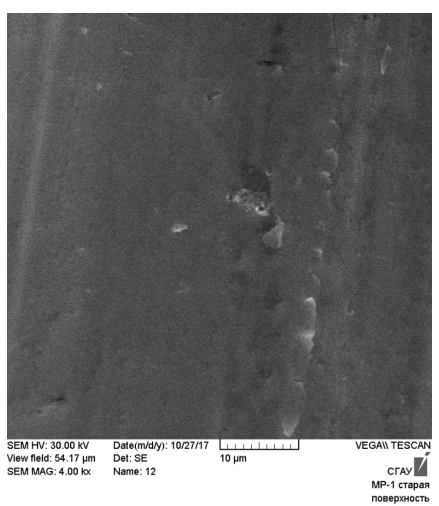

a разцов при травлении проявлялось в виде более тёмного оттенка поверхности. При последующем оксидировании образцов при разных температурах цвет поверхности становился также более тёмным: чем больше температура, тем темнее.

В результате количественного анализа экстракта ДОФ в ИПС, полученного путём абсорбционного концентрирования [10], определили ориентировочную концентрацию ДОФ в газовой фазе $\mathrm{C}=0.46 \pm 0.15 \quad \mathrm{мг} / \mathrm{M}^{3} \quad(\mathrm{n}=3, \quad \mathrm{P}=0.95) \quad$ при описанных выше условиях получения газового потока, содержащим микропримеси ДОФ. Таким образом, для обеспечения определения ДОФ на уровне $0.5 \mathrm{мг} / \mathrm{M}^{3}$ необходимо отобрать объем

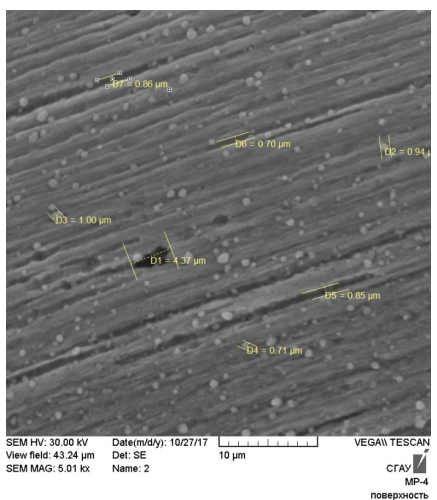

б

Рис. 3. Электронные изображения поверхности образцов блочного материал с варьируемой порозностью:, полученные с использованием сканирующего электронного микроскопа TESCAN «Vega SB»: a - с необработанной поверхностью, б - с поверхностью, подвергнутой травлению и оксидированию при $500^{\circ} \mathrm{C}$.

Fig. 3 Electronic images of surfaces of samples of the block material with variable porosity obtained using a TESCAN "Vega SB" scanning electron microscope: a - with untreated surface, $\mathrm{b}$ - with a surface subjected to etching and oxidation at $500^{\circ} \mathrm{C}$ 
Таблица 1 Зависимость массы десорбированного ДОФ от типа экспериментального образца

Table 1 Dependence of the mass of desorbed DOP on the type of experimental sample

\begin{tabular}{|c|c|c|}
\hline Образец & Способ обработки поверхности & $\begin{array}{c}\text { Масса ДОФ } \\
\mathrm{m} \pm \Delta(\mathrm{n}=3, \mathrm{P}=0,95), \text { мкг }\end{array}$ \\
\hline 1 & без обработки & $15 \pm 6$ \\
\hline 2 & травление & $34 \pm 6$ \\
\hline 3 & травление и оксидирование при $350^{\circ} \mathrm{C}$ & $33 \pm 5$ \\
\hline 4 & травление и оксидирование при $500^{\circ} \mathrm{C}$ & $31 \pm 5$ \\
\hline 5 & травление и оксидирование при $750^{\circ} \mathrm{C}$ & $28 \pm 7$ \\
\hline
\end{tabular}

воздуха в размере 20 дм $^{3}$, т.е. при скорости газового потока $v=0.5 \mathrm{gm}^{3} /$ мин оптимальное время отбора пробы должно составлять 40 минут.

Масса ДОФ, десорбированного с образцов сорбционных систем при описанных выше условиях, представлена в таблице 1.

Установлено, что наименьшее количество ДОФ десорбировано с необработанного образца. Это связано с тем, что данный образец практически не обладает высокоразвитым адсорбционным слоем. Количество десорбированного ДОФ с образцов, обработанных при разных температурах, отличается незначительно. При этом масса ДОФ у образца, обработанного соляной кислотой, но не оксидированного, сопоставима с остальными. Масса десорбированного ДОФ с образцов 2-5 составили в среднем $34 \pm 6$ мкг $(\mathrm{n}=3, \mathrm{P}=0.95)$, т.е. отличие находится в пределах погрешности определения. Видимо, это связано с тем, что ключевым фактором, влияющим на сорбционные свойства изготовленных сорбционных систем, является удельная площадь поверхности, а не ее химический состав. Задача стадии травления заключается в растворении плотной оксидной пленки, которая образовалась на поверхности проволоки в результате ее изготовления и формировании пористой высокоразвитой поверхности.

В рамках работы проведена сравнительная оценка различных способов концентрирования, результаты которой представлены в табл. 2. При этом варьировали объем отбираемой газовой смеси путем изменения времени пропускания потока через сорбционную систему.

Установлено, что стандартизированная методика определения ДОФ с абсорбционным концентрированием [9] дает явно заниженные результаты по сравнению с адсорбционным концентрированием. Это может быть связано как с неполным улавливанием ДОФ из газового потока, так и с внесением дополнительных погрешностей при упаривании экстракта. Стоит отметить, что при pеализации абсорбционного улавливания относительная случайная погрешность в 1.5-2 раза больше, чем при использовании полученных сорбционных систем. Таким образом, использование предлагаемых систем позволяет повысить не только правильность определения ДОФ за счет более эффективного улавливания компонента, но и прецизионность анали3a.

Адсорбционное концентрирование с использованием активированного угля также даёт заниженный результат. Десорбция ДОФ с активированного угля проводилась также, как и в случае с полученными экспериментальными образцами. Заниженный результат может быть связан с неполнотой десорбции ДОФ с активированного угля: при повторной десорбции ДОФ с активированного угля концентрация ДОФ в растворителе была сопоставима с первой десорбцией.

Также установлено, что для проведения концентрирования с использованием предлагаемых сорбционных систем достаточно отобрать 5-10 литров газовой 
Таблица 2. Определенная концентрация ДОФ в газовом потоке при проведении абсорбционного и адсорбционного концентрирования

Table 2. Determined concentration of DOP in the gas flow during the absorption and adsorption concentrations

\begin{tabular}{|c|c|c|c|}
\hline \multirow[t]{2}{*}{ Тип концентрирования } & \multicolumn{3}{|c|}{$\begin{array}{c}\text { Концентрация ДОФ } \mathrm{C} \pm \Delta(\mathrm{n}=3, \mathrm{P}=0.95), \mathrm{M \Gamma} / \mathrm{m}^{3}, \\
\text { при различных объемах газовой фазы, отобранной } \\
\text { для анализа }\end{array}$} \\
\hline & $\mathrm{V}_{\Gamma \Phi}=20$ дм $^{3}$ & $\mathrm{~V}_{\Gamma \Phi}=10$ дм $^{3}$ & $\mathrm{~V}_{\Gamma \Phi}=5$ дм $^{3}$ \\
\hline Абсорбционное концентрирование & $0.46 \pm 0.15$ & $0.14 \pm 0.06$ & $\begin{array}{c}\text { менее предела } \\
\text { обнаружения }\end{array}$ \\
\hline $\begin{array}{c}\text { Адсорбционное концентрирование с } \\
\text { использованием активированного } \\
\text { угля БАУ }\end{array}$ & $0.12 \pm 0.05$ & $\begin{array}{c}\text { менее предела } \\
\text { обнаружения }\end{array}$ & $\begin{array}{c}\text { менее предела } \\
\text { обнаружения }\end{array}$ \\
\hline $\begin{array}{l}\text { Адсорбционное концентрирование с } \\
\text { использованием сорбционной систе- } \\
\text { мы с необработанной поверхностью }\end{array}$ & $0.8 \pm 0.3$ & $1.2 \pm 0.5$ & $1.3 \pm 0.5$ \\
\hline $\begin{array}{c}\text { Адсорбционное концентрирование с } \\
\text { использованием сорбционной систе- } \\
\text { мы с травленной поверхностью }\end{array}$ & $1.7 \pm 0.3$ & $2.2 \pm 0.4$ & $2.2 \pm 0.3$ \\
\hline $\begin{array}{c}\text { Адсорбционное концентрирование с } \\
\text { использованием сорбционной систе- } \\
\text { мы с травленной и оксидированной } \\
\text { при } 350^{\circ} \mathrm{C} \text { поверхностью } \\
\end{array}$ & $1.7 \pm 0.3$ & $2.3 \pm 0.3$ & $2.2 \pm 0.3$ \\
\hline $\begin{array}{c}\text { Адсорбционное концентрирование с } \\
\text { использованием сорбционной систе- } \\
\text { мы с травленной и оксидированной } \\
\text { при } 500^{\circ} \mathrm{C} \text { поверхностью } \\
\end{array}$ & $1.6 \pm 0.3$ & $2.2 \pm 0.5$ & $2.4 \pm 0.3$ \\
\hline $\begin{array}{c}\text { Адсорбционное концентрирование с } \\
\text { использованием сорбционной систе- } \\
\text { мы с травленной и оксидированной } \\
\text { при } 750^{\circ} \mathrm{C} \text { поверхностью }\end{array}$ & $1.4 \pm 0.4$ & $1.9 \pm 0.4$ & $1.8 \pm 0.4$ \\
\hline
\end{tabular}

фазы: при увеличении объема отобранной газовой фазы наблюдается уменьшение определенной концентрации ДОФ, что обусловлено неполным улавливанием компонента из пропускаемого потока.

\section{Заключение}

В рамках работы были изготовлены экспериментальные образцы сорбционных систем с варьируемой порозностью для концентрирования высококипящих органических соединений.

Установлено, что наилучшим сорбционными свойствами по отношению к ДОФ обладают образцы, подвергнутые травлению и оксидированию, причем при данных условиях проведения концентрирования наличие стадии оксидирования при изготовлении образцов существенно не влияет на их сорбционные свойства. Экспериментально установлено, что определение ДОФ с использованием абсорбционного концентрирования и адсорбционного концентрирования с использованием активированного угля дает заниженные результаты по сравнению с адсорбционным концентрированием с использованием полученных сорбционных систем. Применение предлагаемых сорбционных систем позволяет уменьшить время пробоотбора, повысить правильность и прецизионность анализа. 


\section{Список литературы}

1. Иголкин А.А., Сафин А.И., Шахматов Е.В. // Судостроение. 2012. Т. 8. № 4. С. 4648.

2. Сафин А.И., Иголкин А.А., Шахматов Е.В. // Вектор науки ТГУ. 2013. № 2. С. 200202.

3. Тупикова Е.Н. Дисс. канд. хим. наук. Самара. 2003. $153 \mathrm{c}$.

4. Федоров А.А., Казиев Г.З., Казакова Г.Д. Методы химического анализа объектов природной среды. М. КолосС. 2013. 118 с.

5. Mark. D., Robertson D.J. // J. Air Pollut. Control. Assoc. 1990. Vol. 30. pp. 261-267.
6. Другов Ю.С., Родин А.А. Газохроматографический анализ загрязненного воздуха. М. Бином. Лаборатория знаний. 2015. 531 с.

7. Платонов И.А., Колесниченко И.Н., Новикова Е.А. // Сорбиионные и хроматографические процессы. 2017. Т. 17. № 3. С. 378387.

8. Platonov I.A., Rodinkov O.V., Gorbacheva A.R. // Journal of Analytical Chemistry. 2018. Vol. 73. pp. 109-127.

9. МУК 4.1.3168-14. М.: Федеральный центр гигиены и эпидемиологии Роспотребнадзора, 2015. $30 \mathrm{c}$.

10. MУ 5222-90. M. 1990. 15 c.

\title{
The determination of high-boiling organic compounds using block-porous sorption systems
}

\author{
(C) 2021 Platonov I.A., Novikova E.A., Karsunkina A.S., \\ Lebedev A.N., Tupikova E.N.
}

Korolev Samara National Research University, Samara

\begin{abstract}
The determination of trace amounts of toxic substances in atmospheric air (with a concentration of $0.1-0.5 \mathrm{mg} / \mathrm{m}^{3}$ ) is hindered during the stage of concentration, which is one of the main sources of error. Concentration is carried out using sorbents of different natures. The block material with variable porosity developed at Samara University (formerly Kuibyshev Aviation Institute) has advantages over other sorbents due to the possibility to pass large volumes of air through it and due to the simple desorption process.

The aim of the work was to study the possibility of using a block material with a variable porosity as a foundation for a sorption system designed to concentrate high-boiling organic compounds.

$\mathrm{X} 18 \mathrm{H} 10 \mathrm{~T}$ steel was used to manufacture experimental samples. The process of samples production included the stages of forming base blocks and etching samples in hydrochloric acid followed by their oxidation at temperatures of 350,500 , and $750^{\circ} \mathrm{C}$ (the colour of the surface became darker: the higher the temperature, the darker the colour). The study of the obtained samples was carried out using the example of identifying a high-boiling compound, dioctylphthalate (DOP). To study the obtained samples, as well as to evaluate and compare the absorption (the concentration system of an absorber with isopropyl alcohol) and adsorption (the concentration system of a silicone tube with a sample of the sorption system) concentration, an installation was assembled used to produce gas flows with trace amounts of DOP in the air. The quantitative analysis of the obtained concentrates was conducted by gas chromatography.

As a result of the quantitative analysis of the DOP extract in isopropyl alcohol (IPA) obtained by absorption concentration, the approximate concentration of DOP in the gas phase was determined $\mathrm{C}=0.46 \pm$ $0.15 \mathrm{mg} / \mathrm{m}^{3}$. It was found that the smallest amount of DOP was desorbed from the untreated sample. This is due to the fact that this sample practically did not have a highly developed adsorption layer. The amount of DOP desorbed from samples treated at different temperatures differed insignificantly. As part of the work, a comparative assessment of various concentration methods was carried out. It was found that the standardised method to determine DOP with absorption concentration provided clearly underestimated results in comparison to the adsorption concentration. This may be explained by both the incomplete capture of DOP in the gas flow and the introduction of additional errors due to extract evaporation. Adsorption concentration using activated carbon also provided an underestimated result. DOP desorption from activated carbon was carried out similar to the obtained experimental samples. The underestimated result may be associated with incomplete DOP desorption from activated carbon. Thus, using the proposed systems allows increasing not only the accuracy of DOP determination due to the more efficient capture of the component but also the precision of the analysis.
\end{abstract}

Keywords: sorption concentration, high-boiling organic compounds, sample preparation, study of gas environment, chromatographic method of analysis, block-porous systems. 


\section{References}

1. Igolkin A.A., Safin A.I., Shakhmatov E.V., Shipbuilding, 2012, Vol. 8, No 4, pp. 46-48.

2. Safin A.I., Igolkin A.A., Shakhmatov E.V., Vector of Science of TSU, 2013, No 2, pp. 200202.

3. Tupikova E.N. Diss. cand. chem. nauk. Samara, 2003, 153 p.

4. Fedorov A.A., Kaziev G.Z., Kazakova G.D. Methods for chemical analysis of natural environment objects. Moscow, KolosS, 2013, 118 p.

5. Mark. D., Robertson D.J., J. Air Pollut. Control. Assoc, 1990, Vol. 30, pp. 261-267.

Платонов Игорь Артемьевич - профессор кафедры химии, д.т.н., Самарский национальный исследовательский университет им. академика С.П. Королева, Самара

Новикова Екатерина Анатольевна - доцент кафедры химии, к.Х.н., Самарский национальный исследовательский университет им. академика С.П. Королева, Самара

Карсункина Алеся Сергеевна - аспирант кафедры химии, Самарский национальный исследовательский университет им. академика С.П. Королева, Самара

Тупикова Елена Николаевна - доцент кафедры химии, к.х.н., Самарский национальный исследовательский университет им. академика С.П. Королева, Самара

Лебедев Алексей Николаевич - аспирант кафедры физики, Самарский национальный исследовательский университет им. академика С.П. Королева, Самара
6. Drugov Yu.S., Rodin A.A. Gas chromatographic analysis of polluted air. M., Binom. Knowledge laboratory, 2015, $531 \mathrm{p}$.

7. Platonov I.A., Kolesnichenko I.N., Novikova E.A., Sorptsionnye I Khromatograficheskiye protsessy, 2017, Vol. 17, No 3, pp. 378-387.

8. Platonov I.A., Rodinkov O.V., Gorbacheva A.R., Journal of Analytical Chemistry, 2018, Vol. 73, pp. 109-127.

9. MUK 4.1.3168-14. M.: Federal Center for Hygiene and Epidemiology of Rospotrebnadzor, $2015,30 \mathrm{p}$.

10. MU 5222-90. M. 1990, 15 p.

Platonov Igor A. - prof., grand Ph.D (technics), department of Chemistry, Samara National Research University named after academician S.P. Korolev, Samara, e-mail: pia@ssau.ru

Novikova Ekaterina A. - Ph.D. (chemistry), associate prof., department of Chemistry, Samara National Research University named after academician S.P. Korolev, Samara, e-mail: ekanno85@mail.ru

Karsunkina Alesya S. - the postgraduate student of the Department of Chemistry, Samara National Research University named after academician S.P. Korolev, Samara, e-mail: karsunkina.alesya@mail.ru

Tupikova Elena N. - Ph.D. (chemistry), associate prof., department of Chemistry, Samara National Research University named after academician S.P. Korolev, Samara

Lebedev Alexey N. - the postgraduate student of the Department of Physics, Samara National Research University named after academician S.P. Korolev, Samara 